\title{
$N$-Heterocyclic carbene-catalyzed domino hydroacylation/Stetter reactions of salicyl alkynylphosphonates and aromatic aldehydes
}

\author{
Zhen-Qiang Wang, Ying Wang, and De-Qing Shi* \\ Key Laboratory of Pesticide and Chemical Biology of Ministry of Education, College of \\ Chemistry, Central China Normal University, Wuhan 430 079, P. R. China \\ E-mail: chshidq@mail.ccnu.edu.cn
}

\begin{abstract}
1,4-Dicarbonyl compounds 2 carrying a phosphonate group were obtained by utilizing a dually $\mathrm{N}$-heterocyclic carbene (NHC) catalyzed domino reaction involving the intramolecular hydroacylation of salicyl alkynylphosphonates $\mathbf{1}$ and a subsequent intermolecular Stetter reaction with aromatic aldehyde at room temperature. In addition, $\mathbf{2}$ can also be applied for the synthesis of benzopyranopyrrole carrying a phosphonate group $\mathbf{4}$ in one pot and in moderate yield.
\end{abstract}

Keywords: $N$-Heterocyclic carbene; domino reaction; hydroacylation; Stetter reaction; 1,4-dicarbonyl compound; phosphonate

\section{Introduction}

During the past decades, domino reactions, which can rapidly form complicated molecule skeletons from readily available substrates in a single operation without isolation of intermediates, have received more and more attention of organic chemists. So, considerable efforts have been made to develop organocatalytic domino transformations. ${ }^{1}$ 1,4-Dicarbonyl compounds are versatile intermediates in the synthesis of cyclopentenones ${ }^{2}$ and a variety of heterocycles such as furan, ${ }^{3}$ pyrrole, ${ }^{4}$ thiophene, ${ }^{5}$ and pyrrolidine. ${ }^{6}$ Recently, $N$-heterocyclic carbenes (NHCs) have been widely used, not only as ligands in organometallic chemistry, but also as efficient nucleophilic organocatalysts in a variety of organic reactions, such as benzoin condensation, Stetter reaction, $\mathrm{a}^{3}$ to $\mathrm{d}^{3}$ umpolung, and so on, so the development of NHCs in organic synthesis has attracted more and more attention. ${ }^{7}$ Glorius and coworkers have pioneered the development of a powerful NHC-catalyzed hydroacylation of unactivated alkynes leaded to the formation of $\alpha, \beta$-unsaturated chromanones, ${ }^{8}$ while intramolecular hydroacylation reactions of activated alkynes were reported recently. ${ }^{9}$

Recently, an effective $N$-heterocyclic carbene catalyzed intramolecular hydroacylation reaction of salicyl alkynylphosphonates was reported in our group, in which both exocyclic and 
endocyclic tautomers can be obtained at different temperatures. ${ }^{9 \mathrm{~b}}$ Because $\alpha, \beta$-unsaturated ketones (exocyclic tautomers) are the major products at lower temperatures $\left(15-17^{\circ} \mathrm{C}\right)$, this prompted us to investigate the possibility of developing a dually NHC-catalyzed domino reaction involving the intramolecular hydroacylation of salicyl alkynylphosphonates and a subsequent intermolecular Stetter reaction to prepare 1,4-dicarbonyl compounds 2 carrying a phosphonate group at room temperature (Scheme 2), which can be very important intermediates for the synthesis of biologically active phosphonyl heterocyclic compounds. ${ }^{10}$ Although Glorius and coworkers reported the NHC-catalyzed cascade hydroacylation/Stetter reactions of salicyl alkynes, ${ }^{8}$ the groups linking with alkynes are usually $\mathrm{H}$ or a Me group, other groups such as aromatic group, ester group linking with alkynes are not reported. As for activated alkynes, no domino hydroacylation/Stetter reaction is reported because in which endocyclic tautomers are the major product. In this paper, we report the first cascade hydroacylation/ Stetter reaction of activated alkynes $\mathbf{1}$ containing a phosphonyl group derived from substituted salicylaldehydes (Scheme 1).

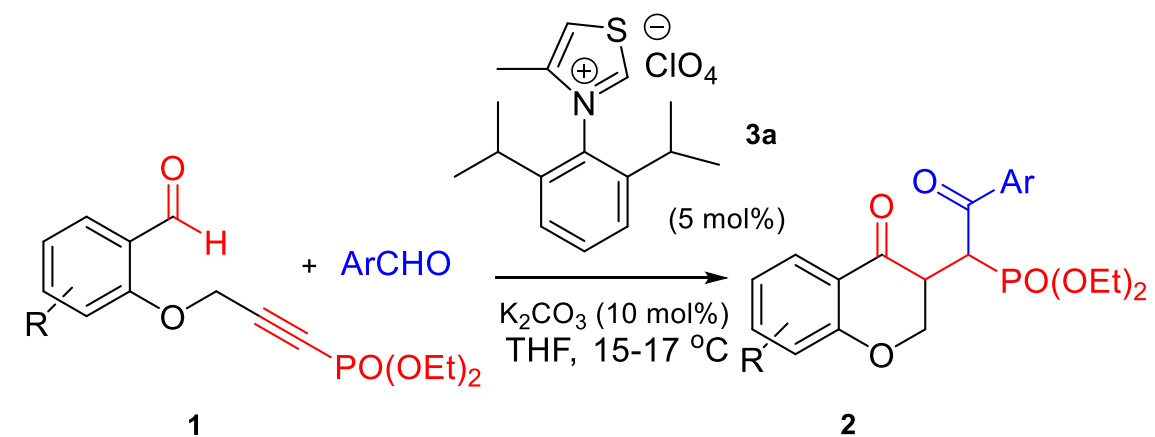

Scheme 1. Domino hydroacylation/Stetter reaction of activated alkynes containing a phosphonyl group.

\section{Results and Discussion}

First of all, we used substrate 1a and 4-chlorobenzaldehyde as a model in order to optimize the reaction condition. The initial screening was carried out at $15-17{ }^{\circ} \mathrm{C}$ with $5 \mathrm{~mol} \%$ of catalyst and $10 \mathrm{~mol} \%$ of $\mathrm{K}_{2} \mathrm{CO}_{3}$ in THF (Table 1, entries 1-6). When thiazolium salt 3a was used as the catalyst and $\mathrm{K}_{2} \mathrm{CO}_{3}$ as the base, $\mathbf{2 a}$ was obtained in $81 \%$ yield (Table1, entry 1), and $\mathbf{3 a}$ is the most effective catalyst, however, the triazolium salt $\mathbf{3 d}$ is not so effective (Table1, entry 4); Secondly, the effects of various bases (Table 1, entries 7-11) were explored using catalyst 3a as a control, the moderate base $\left(\mathrm{K}_{2} \mathrm{CO}_{3}\right)$ afforded the products in higher yields than other bases. Subsequently, we turned our attention to the influence of different solvents on the reaction (Table 1, entries 12-17) and found THF to be the most effective, the more polar EtOH and DMF being less so. Different temperatures have some obvious effects on the reaction. The lower 
temperature $\left(15-17^{\circ} \mathrm{C}\right)$ is more beneficial to the reaction than the higher one $\left(30-35{ }^{\circ} \mathrm{C}\right)$; when the reaction with $\mathbf{3 a}$ and $\mathrm{K}_{2} \mathrm{CO}_{3}$ in THF was tested at $30-35{ }^{\circ} \mathrm{C}$, the yield of $\mathbf{2 a}$ is only $32 \%$. Finally, the optimized conditions were established as following: thiazolium salt $\mathbf{3 a}$ as the pre-catalyst $(5 \mathrm{~mol} \%), \mathrm{K}_{2} \mathrm{CO}_{3}(10 \mathrm{~mol} \%)$ as the base, THF as the solvent, and stirring at $15-17{ }^{\circ} \mathrm{C}$ for $3 \mathrm{~h}$.

Table 1 Optimization of the reaction conditions ${ }^{a}$

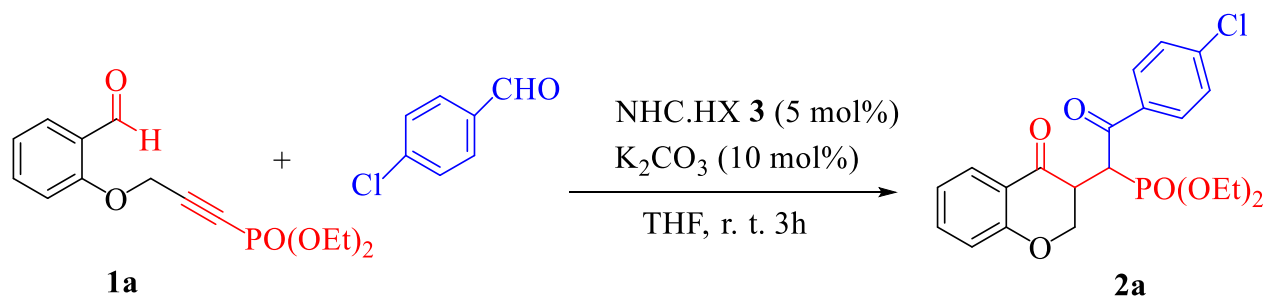<smiles></smiles>

3a
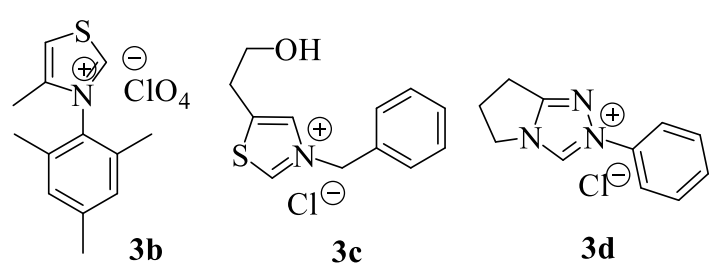

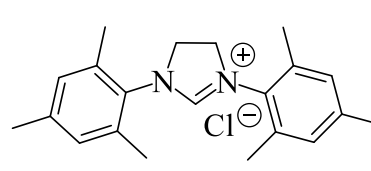

$3 e$

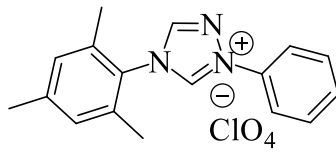

3f

\begin{tabular}{ccccc}
\hline Entry & NHC-HX & Base & Solvent & Yield of 2a [\% $]^{b}$ \\
\hline 1 & $\mathbf{3 a}$ & $\mathrm{K}_{2} \mathrm{CO}_{3}$ & $\mathrm{THF}$ & 81 \\
2 & $\mathbf{3 b}$ & $\mathrm{K}_{2} \mathrm{CO}_{3}$ & $\mathrm{THF}$ & 75 \\
3 & $\mathbf{3 c}$ & $\mathrm{K}_{2} \mathrm{CO}_{3}$ & $\mathrm{THF}$ & 70 \\
4 & $\mathbf{3 d}$ & $\mathrm{K}_{2} \mathrm{CO}_{3}$ & $\mathrm{THF}$ & 20 \\
5 & $\mathbf{3 e}$ & $\mathrm{K}_{2} \mathrm{CO}_{3}$ & $\mathrm{THF}$ & 34 \\
6 & $\mathbf{3 f}$ & $\mathrm{K}_{2} \mathrm{CO}_{3}$ & $\mathrm{THF}$ & 57 \\
7 & $\mathbf{3 a}$ & $\mathrm{Cs} 2 \mathrm{CO}_{3}$ & 65 \\
8 & $\mathbf{3 a}$ & $\mathrm{DBU}$ & 40 \\
9 & $\mathbf{3 a}$ & $\mathrm{DABCO}$ & 38 \\
10 & $\mathbf{3 a}$ & $\mathrm{NaH}$ & $\mathrm{THF}$ & 71 \\
11 & $\mathbf{3 a}$ & $\mathrm{NaOH}_{2}$ & $\mathrm{THF}$ & 58 \\
12 & $\mathbf{3 a}$ & $\mathrm{K}_{2} \mathrm{CO}_{3}$ & Dioxane & 72 \\
13 & $\mathbf{3 a}$ & $\mathrm{K}_{2} \mathrm{CO}_{3}$ & EtOH & 40 \\
14 & $\mathbf{3 a}$ & $\mathrm{K}_{2} \mathrm{CO}_{3}$ & Toluene & 65 \\
15 & $\mathbf{3 a}$ & $\mathrm{K}_{2} \mathrm{CO}_{3}$ & $\mathrm{CH}{ }_{3} \mathrm{CN}$ & 68 \\
16 & $\mathbf{3 a}$ & $\mathrm{K}_{2} \mathrm{CO}_{3}$ & DMF & 30 \\
17 & $\mathbf{3 a}$ & $\mathrm{K}_{2} \mathrm{CO}_{3}$ & DCM & 76 \\
\hline
\end{tabular}

${ }^{a}$ General conditions: 1a $(0.5 \mathrm{mmol})$, NHC-HX $(5 \mathrm{~mol} \%)$, base $(10 \mathrm{~mol} \%)$, solvent $(2.0 \mathrm{ml})$, room temperature $\left(15-17^{\circ} \mathrm{C}\right), 3 \mathrm{~h} ; \quad{ }^{b}$ Isolated yields. 
With the optimised conditions thus established, we tested different substrates to give good results in the NHC catalyzed cascade reaction of hydroacylation and Stetter reaction. For salicyl alkynylphosphonates with both electron-donating groups and electron-withdrawing ones on the phenyl ring, the reactions proceeded smoothly and gave the corresponding $\mathbf{2}$ in good yields at room temperature. However, when benzaldehyde was used, the yield was very low (Table 2, entry 7); the reason for this is not clear.

Table 2 Scope of the domino hydroacylation/Stetter reactions. ${ }^{a}$

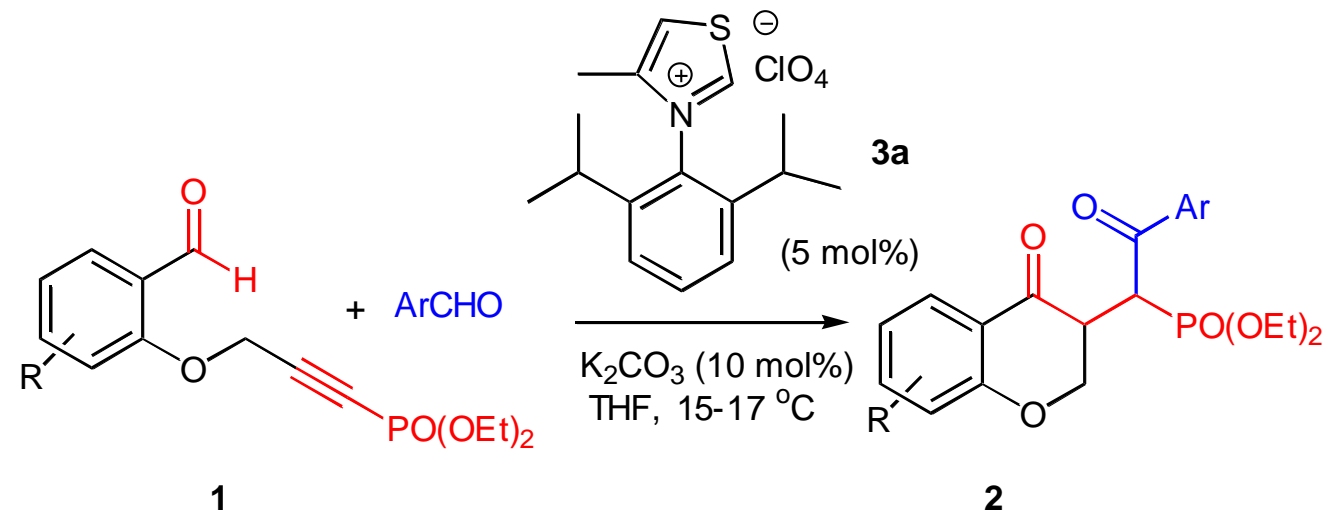

\begin{tabular}{cccc}
\hline Entry & $\mathrm{R}$ & $\mathrm{Ar}$ & Yield of 2 [\%] $^{b}$ \\
\hline 1 & $\mathrm{H}(\mathbf{1 a})$ & $4-\mathrm{ClC}_{6} \mathrm{H}_{4}$ & $81(\mathbf{2 a})$ \\
2 & $4-\mathrm{MeO}(\mathbf{1 b})$ & $4-\mathrm{BrC}_{6} \mathrm{H}_{4}$ & $84(\mathbf{2 b})$ \\
3 & $4-\mathrm{F} \mathrm{(1c)}$ & $4-\mathrm{BrC}_{6} \mathrm{H}_{4}$ & $83(\mathbf{2 c})$ \\
4 & $4-t-\mathrm{Bu}(\mathbf{1 d})$ & $4-\mathrm{BrC}_{6} \mathrm{H}_{4}$ & $71(\mathbf{2 d})$ \\
5 & $6-\mathrm{Me} \mathrm{(1e)}$ & $4-\mathrm{BrC}_{6} \mathrm{H}_{4}$ & $77(\mathbf{2 e})$ \\
6 & $4,6-\mathrm{di}-t$-Bu (1) & $4-\mathrm{BrC}_{6} \mathrm{H}_{4}$ & $69(\mathbf{2 f})$ \\
7 & $\mathrm{H} \mathrm{(1a)}$ & $\mathrm{C}_{6} \mathrm{H}_{5}$ & $<5$ \\
\hline
\end{tabular}

${ }^{a}$ General conditions: 1 (0.5 mmol, 1.0 equiv), $3 \mathbf{a}(0.025 \mathrm{mmol}, 5 \mathrm{~mol} \%), \mathrm{K}_{2} \mathrm{CO}_{3}(0.05 \mathrm{mmol}, 10 \mathrm{~mol} \%)$, $\operatorname{ArCHO}\left(0.5 \mathrm{mmol}, 1.0\right.$ equiv), THF $(2.0 \mathrm{ml}), 15-17^{\circ} \mathrm{C}, 3 \mathrm{~h} ; \quad{ }^{b}$ Isolated yields

In order to prepare novel heterocycles containing a phosphonyl group with potent biological activities, $\mathbf{2}$ can be applied to the synthesis of benzopyranopyrrolephosphonate $\mathbf{4}$ successfully via a one-pot reaction in $50 \%$ yield (Scheme 2). 


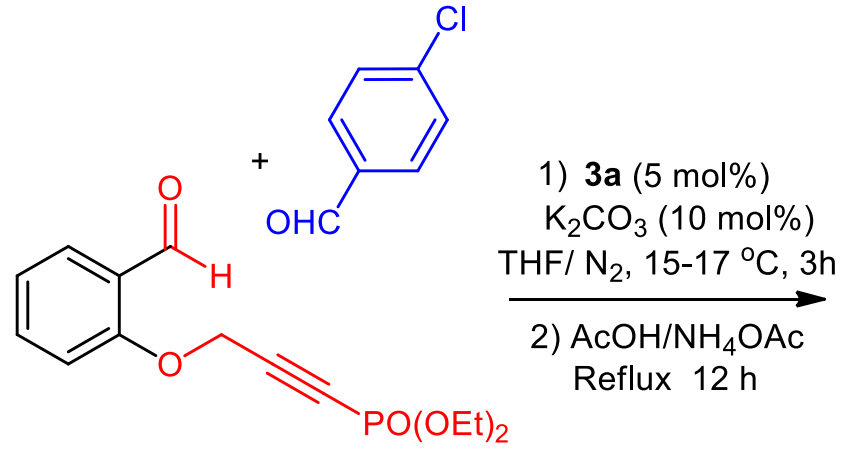

$1 \mathrm{a}$

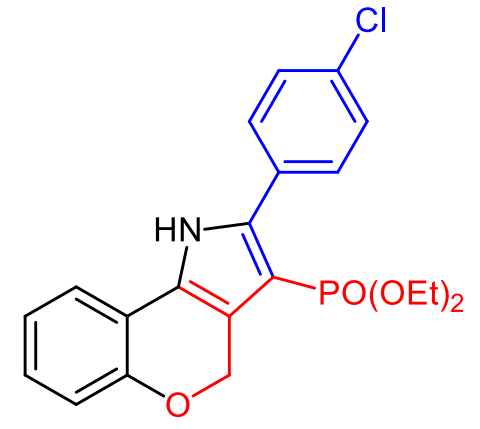

4

Scheme 2 Preparation of the benzopyranopyrrolephosphonate 4 by a one-pot reaction.

\section{Conclusions}

In conclusion, 1,4-dicarbonyl compounds 2 carrying a phosphonate group were obtained by utilizing a dually NHC-catalyzed domino reaction involving the intramolecular hydroacylation of salicyl alkynylphosphonates $\mathbf{1}$ and a subsequent intermolecular Stetter reaction with aromatic aldehyde in good yields at room temperature. In addition, $\mathbf{2}$ can be also applied successfully for the synthesis of benzopyranopyrrole containing a phosphonyl group $\mathbf{4}$ in one-pot reaction in moderate yield.

\section{Experimental Section}

General. All reagents and solvents for reactions were used as received with the following exceptions. THF, toluene and dioxane were distilled from $\mathrm{Na} /$ benzophenone. Dichloromethane, acetonitrile and $N, N$-dimethylformamide (DMF) were distilled from calcium hydride. Ethanol and $n$-butyl alcohol were distilled form $\mathrm{Mg}$ turnings. $\mathrm{K}_{2} \mathrm{CO}_{3}$ was dried by heating at $110{ }^{\circ} \mathrm{C}$ for $12 \mathrm{~h}$ and left to cool under Ar. $\mathrm{N}$-Heterocyclic carbene (NHC) precursors 3 were prepared according to the literature. ${ }^{11}$ The substituted salicyl alkynylphosphonates $\mathbf{1}$ were synthesized according to the the reported method. ${ }^{9 \mathrm{~b}}$ All other commercially available solvents and reagents were used without further purification. All reactions were performed in oven-dried apparatus under $\mathrm{N}_{2}$ or Ar atmosphere. Reactions were monitored by thin layer chromatography (TLC) and visualized by UV light or $\mathrm{KMnO}_{4}$ staining solution followed by heating.

Melting points were determined with a WRS-1B digital melting point apparatus. ${ }^{1} \mathrm{H} \mathrm{NMR},{ }^{13} \mathrm{C}$ NMR spectra and ${ }^{31} \mathrm{P}$ NMR spectra were obtained with a Varian Mercury PLUS 600 spectrometer. Chemical shift in ppm, internal standard TMS or $\mathrm{CHCl}_{3}$, multiplicity ( $\mathrm{s}=$ singlet, $\mathrm{d}$ = doublet, $\mathrm{t}=$ triplet, $\mathrm{q}=$ quartet, and $\mathrm{m}=$ multiplet ), coupling constants $\mathrm{Hz} .{ }^{13} \mathrm{C} \mathrm{NMR}$ chemical shifts are reported in ppm from $\mathrm{CDCl}_{3}$ (taken as $77.0 \mathrm{ppm}$ ). ${ }^{31} \mathrm{P}$ NMR spectra were recorded with 
$85 \% \mathrm{H}_{3} \mathrm{PO}_{4}$ as external standard. The mass spectra (ESI) were obtained using an Applied Biosystems API 2000 LC/MS/MS (ESI-MS) spectrometer. Elemental analyses were performed with an Elementar Vario ELW CHNSO elemental analyzer.

Synthesis of 2-phosphonyl 1,4-diketones 2: general procedure. To an oven-dried round-bottomed flask equipped with a magnetic stirring bar was added diethyl 3-(2-formylphenoxy)prop-1-ynylphosphonate 1 ( $0.5 \mathrm{mmol}, 1.0$ equiv), pre-catalyst $\mathbf{3 a}$ (9 mg, $0.025 \mathrm{mmol}, 0.05$ equiv), substituted benzaldehyde ( $0.5 \mathrm{mmol}, 1.0$ equiv), dry THF ( $2 \mathrm{ml})$ and dry $\mathrm{K}_{2} \mathrm{CO}_{3}$ ( $6.9 \mathrm{mg}, 0.05 \mathrm{mmol}, 0.10$ equiv) under a $\mathrm{N}_{2}$ or Ar atmosphere at $15-17{ }^{\circ} \mathrm{C}$. The resulting mixture was then stirred for $3 \mathrm{~h}$ at the same temperature. The progress of the reaction was monitored by TLC. Upon completion, the mixture was pre-absorbed on silica gel and purified by flash column chromatography on silica gel (eluent: hexane/ AcOEt 1:1) to afford 2 as a white solid.

Diethyl 2-(4-chlorophenyl)-1-(4-oxochroman-3-yl)-2-oxoethylphosphonate (2a). Prepared according to the general procedure. The product was obtained as a white solid ( $81 \%$ yield), mp 102-104 ${ }^{\circ} \mathrm{C} .{ }^{1} \mathrm{H}$ NMR $\left(600 \mathrm{MHz}, \mathrm{CDCl}_{3}\right): \delta 8.01(\mathrm{~d}, J 8.4 \mathrm{~Hz}, 2 \mathrm{H}), \delta 7.75(\mathrm{~d}, J 7.8 \mathrm{~Hz}, 1 \mathrm{H}), \delta$ $7.49(\mathrm{~d}, J 7.8 \mathrm{~Hz}, 3 \mathrm{H}), \delta 6.98(\mathrm{~d}, J 8.4 \mathrm{~Hz}, 2 \mathrm{H}), \delta 5.17(\mathrm{dd}, J 15.0 \mathrm{~Hz}, J 5.4 \mathrm{~Hz}, 1 \mathrm{H}), \delta 4.38-4.43$ $(\mathrm{m}, 2 \mathrm{H}), \delta 4.01-4.16(\mathrm{~m}, 5 \mathrm{H}), \delta 1.28(\mathrm{t}, J 7.2 \mathrm{~Hz}, 3 \mathrm{H}), \delta 1.17(\mathrm{t}, J 7.2 \mathrm{~Hz}, 3 \mathrm{H}) .{ }^{13} \mathrm{C}$ NMR $(150$ $\left.\mathrm{MHz}, \mathrm{CDCl}_{3}\right): \delta 193.3,192.2,161.5,139.4,136.3,135.6,130.2,128.8,127.3,121.5,119.8$, 117.6, 69.6, 63.2, 46.9, 44.3 (d, $J 128.4 \mathrm{~Hz}), 16.1 .{ }^{31} \mathrm{P}$ NMR (243 MHz, $\left.\mathrm{CDCl}_{3}\right): \delta 18.8$. ESI-MS: $m / z 437[\mathrm{M}+\mathrm{H}]^{+}, 459[\mathrm{M}+\mathrm{Na}]^{+}, 475[\mathrm{M}+\mathrm{K}]$. Anal. calcd for $\mathrm{C}_{21} \mathrm{H}_{22} \mathrm{ClO}_{6} \mathrm{P}: \mathrm{C}, 57.74 ; \mathrm{H}, 5.08$. Found: C, 56.83; H, $5.01 \%$.

Diethyl 2-(4-bromophenyl)-1-(6-methoxy-4-oxochroman-3-yl)-2-oxoethylphosphonate (2b). Prepared according to the general procedure. The product was obtained as white solid (84\%), $\mathrm{m}$ p 126-127 ${ }^{\circ} \mathrm{C} .{ }^{1} \mathrm{H}$ NMR $\left(600 \mathrm{MHz}, \mathrm{CDCl}_{3}\right): \delta 7.93(\mathrm{~d}, J 8.4 \mathrm{~Hz}, 2 \mathrm{H}), \delta 7.64(\mathrm{~d}, J 8.4 \mathrm{~Hz}, 2 \mathrm{H}), \delta$ $7.16(\mathrm{~s}, 1 \mathrm{H}), \delta 7.08(\mathrm{~d}, J 9.6 \mathrm{~Hz}, 1 \mathrm{H}), \delta 6.93(\mathrm{~d}, J 9.6 \mathrm{~Hz}, 1 \mathrm{H}), \delta 5.13(\mathrm{dd}, J 11.4 \mathrm{~Hz}, J 4.8 \mathrm{~Hz}$, $1 \mathrm{H}), \delta 4.34-4.39(\mathrm{~m}, 2 \mathrm{H}), \delta 4.03-4.16(\mathrm{~m}, 5 \mathrm{H}), \delta 3.71(\mathrm{~s}, 3 \mathrm{H}), \delta 1.28(\mathrm{t}, J 6.6 \mathrm{~Hz}, 3 \mathrm{H}), \delta 1.18(\mathrm{t}$, $J 6.6 \mathrm{~Hz}, 3 \mathrm{H}) .{ }^{13} \mathrm{C} \mathrm{NMR}\left(150 \mathrm{MHz}, \mathrm{CDCl}_{3}\right): \delta 193.4,192.3,156.3,154.0,136.1,131.8,130.5$, 130.2, 128.2, 125.6, 119.1, 107.3 (d, $J 101.4 \mathrm{~Hz}), 69.7,63.1,55.6,46.9,43.9$ (d, $J 130.4 \mathrm{~Hz})$, 16.1. ${ }^{31} \mathrm{P}$ NMR $\left(243 \mathrm{MHz}, \mathrm{CDCl}_{3}\right): \delta$ 16.4. ESI-MS: $m / z, 512[\mathrm{M}+\mathrm{H}]^{+}, 434[\mathrm{M}+\mathrm{Na}]^{+}, 550$ $[\mathrm{M}+\mathrm{K}]$. Anal. calcd for $\mathrm{C}_{22} \mathrm{H}_{24} \mathrm{BrO}_{7} \mathrm{P}: \mathrm{C}, 51.68 ; \mathrm{H}, 4.73$. Found: C, 51.86; H, $4.84 \%$.

Diethyl 2-(4-bromophenyl)-1-(6-fluoro-4-oxochroman-3-yl)-2-oxoethylphosphonate (2c). Prepared according to the general procedure. The product was obtained as white solid (83\%), $\mathrm{mp}$ 97-99 ${ }^{\circ} \mathrm{C} . \quad{ }^{1} \mathrm{H}$ NMR $\left(600 \mathrm{MHz}, \mathrm{CDCl}_{3}\right): \delta 7.92(\mathrm{~d}, J 8.4 \mathrm{~Hz}, 2 \mathrm{H}), \delta 7.64(\mathrm{~d}, J 8.4 \mathrm{~Hz}, 2 \mathrm{H}), \delta$ 7.39 (dd, J $8.4 \mathrm{~Hz}, J 3.0 \mathrm{~Hz}, 1 \mathrm{H}), \delta 7.20$ (t, J $3.0 \mathrm{~Hz}, 1 \mathrm{H}), \delta 6.98$ (dd, $J 4.2 \mathrm{~Hz}, J 9.0 \mathrm{~Hz}, 1 \mathrm{H}), \delta$ $5.17(\mathrm{dd}, J 11.4 \mathrm{~Hz}, J 5.4 \mathrm{~Hz}, 1 \mathrm{H}), \delta 4.40(\mathrm{~m}, 2 \mathrm{H}), \delta 4.03-4.14(\mathrm{~m}, 5 \mathrm{H}), \delta 1.27(\mathrm{t}, J 7.2 \mathrm{~Hz}, 3 \mathrm{H})$, $\delta 1.18\left(\mathrm{t}, J\right.$ 7.2 Hz, 3H). ${ }^{13} \mathrm{C} \mathrm{NMR}\left(150 \mathrm{MHz}, \mathrm{CDCl}_{3}\right): \delta$ 193.3, 191.3, 161.4, 157.8, 135.9, 131.9, 131.6 (d, J $32 \mathrm{~Hz}), 130.3(\mathrm{~d}, J 30 \mathrm{~Hz}), 128.4,123.9$ (d, J $25 \mathrm{~Hz}), 119.4,112.2$ (d, J $23 \mathrm{~Hz})$, 69.8, $63.3(\mathrm{t}, J 41.2 \mathrm{~Hz}), 46.8,43.8(\mathrm{~d}, J 129 \mathrm{~Hz}), 16.2(\mathrm{~d}, J 16 \mathrm{~Hz}) .{ }^{31} \mathrm{P} \mathrm{NMR}(243 \mathrm{MHz}$, 
$\left.\mathrm{CDCl}_{3}\right): \delta$ 19.5. ESI-MS: $m / z 499[\mathrm{M}+\mathrm{H}]^{+}, 521[\mathrm{M}+\mathrm{Na}]^{+}, 537[\mathrm{M}+\mathrm{K}]$. Anal. calcd for $\mathrm{C}_{21} \mathrm{H}_{21} \mathrm{BrFO}_{6} \mathrm{P}: \mathrm{C}, 50.52 ; \mathrm{H}, 4.24$. Found: $\mathrm{C}, 50.41 ; \mathrm{H}, 4.35 \%$.

Diethyl 2-(4-bromophenyl)-1-(6-tert-butyl-4-oxochroman-3-yl)-2-oxoethylphosphonate (2d). Prepared according to the general procedure. The product was obtained as white solid (71\%), $\mathrm{m}$ p 99-101 ${ }^{\circ} \mathrm{C} . \quad{ }^{1} \mathrm{H} \mathrm{NMR}\left(600 \mathrm{MHz}, \mathrm{CDCl}_{3}\right): \delta 7.95(\mathrm{~d}, J 7.8 \mathrm{~Hz}, 2 \mathrm{H}), 7.74(\mathrm{~s}, 1 \mathrm{H}), \delta 7.65(\mathrm{~d}, J$ $7.8 \mathrm{~Hz}, 2 \mathrm{H}), \delta 7.54(\mathrm{~d}, J 7.2 \mathrm{~Hz}, 1 \mathrm{H}), \delta 6.93(\mathrm{~d}, J 9.0 \mathrm{~Hz}, 1 \mathrm{H}), \delta 5.13$ (dd, J $12.0 \mathrm{~Hz}, J 6.0 \mathrm{~Hz}$, $1 \mathrm{H}), \delta 4.32-4.38(\mathrm{~m}, 2 \mathrm{H}), \delta 4.03-4.15(\mathrm{~m}, 5 \mathrm{H}), \delta 1.29(\mathrm{t}, J 7.2 \mathrm{~Hz}, 3 \mathrm{H}), \delta 1.25(\mathrm{t}, J 7.2 \mathrm{~Hz}, 3 \mathrm{H})$. ${ }^{13} \mathrm{C}$ NMR $\left(150 \mathrm{MHz}, \mathrm{CDCl}_{3}\right): \delta 193.5,192.7,159.5,144.5,136.1,134.2,130.5,128.8,128.2$, 123.4, 119.0, 117.3, 69.6, 63.2, 47.2, 43.5, 34.3, 31.2 (d, $J$ 142.6 Hz), 16.3. ${ }^{31} \mathrm{P}$ NMR (243 MHz, $\left.\mathrm{CDCl}_{3}\right): \delta$ 13.2. ESI-MS: $m / z 537[\mathrm{M}+\mathrm{H}]^{+}, 559[\mathrm{M}+\mathrm{Na}]^{+}, 575[\mathrm{M}+\mathrm{K}]$. Anal. calcd for $\mathrm{C}_{25} \mathrm{H}_{30} \mathrm{BrO}_{6} \mathrm{P}: \mathrm{C}, 55.88$; H, 5.63. Found: C, 55.71; H, $5.57 \%$.

Diethyl 2-(4-bromophenyl)-1-(8-methyl-4-oxochroman-3-yl)-2-oxoethylphosphonate (2e). Prepared according to the general procedure. The product was obtained as white solid (77\%), $\mathrm{m}$ p 109-110 ${ }^{\circ} \mathrm{C} .{ }^{1} \mathrm{H}$ NMR $\left(600 \mathrm{MHz}, \mathrm{CDCl}_{3}\right): \delta 7.93(\mathrm{~d}, J 8.4 \mathrm{~Hz}, 2 \mathrm{H}), \delta 7.65(\mathrm{~d}, J 8.4 \mathrm{~Hz}, 2 \mathrm{H}), \delta$ $7.59(\mathrm{~d}, J 8.4 \mathrm{~Hz}, 1 \mathrm{H}), \delta 7.34(\mathrm{~d}, J 7.2 \mathrm{~Hz}, 1 \mathrm{H}), \delta 6.88(\mathrm{t}, J 7.8 \mathrm{~Hz}, 1 \mathrm{H}), \delta 5.20(\mathrm{dd}, J 11.4 \mathrm{~Hz}, J$ $5.4 \mathrm{~Hz}, 1 \mathrm{H}), \delta 4.36-4.41(\mathrm{~m}, 2 \mathrm{H}), \delta 4.04-4.17(\mathrm{~m}, 5 \mathrm{H}), \delta 1.29(\mathrm{t}, J 7.2 \mathrm{~Hz}, 3 \mathrm{H}), \delta 1.19(\mathrm{t}, J 7.2$ $\mathrm{Hz}, 3 \mathrm{H}) .{ }^{13} \mathrm{C} \mathrm{NMR}\left(150 \mathrm{MHz}, \mathrm{CDCl}_{3}\right): \delta 193.5,192.4,159.8,137.2,136.1,131.8,130.3,128.2$, 127.0, 124.9, 120.9, 119.4, 63.2, 46.9, 44.3, 43.5, 16.2, 15.5. ${ }^{31} \mathrm{P}$ NMR $\left(243 \mathrm{MHz}, \mathrm{CDCl}_{3}\right): \delta$ 15.1. ESI-MS: $m / z 495[\mathrm{M}+\mathrm{H}]^{+}, 517[\mathrm{M}+\mathrm{Na}]^{+}, 533[\mathrm{M}+\mathrm{K}]$. Anal. calcd for $\mathrm{C}_{22} \mathrm{H}_{24} \mathrm{BrO}{ }_{6} \mathrm{P}: \mathrm{C}$, 53.35; H, 4.88. Found: C, 53.19; H, $4.64 \%$.

Diethyl 2-(4-bromophenyl)-1-(6,8-di-tert-butyl-4-oxochroman-3-yl)-2-oxoethylphosphonate (2f). Prepared according to the general procedure. The product was obtained as white solid (69\%), mp 114-116 ${ }^{\circ} \mathrm{C} .{ }^{1} \mathrm{H}$ NMR $\left(600 \mathrm{MHz}, \mathrm{CDCl}_{3}\right): \delta 7.93(\mathrm{~d}, J 8.4 \mathrm{~Hz}, 2 \mathrm{H}), \delta 7.65(\mathrm{~d}, J 8.4$ $\mathrm{Hz}, 2 \mathrm{H}), \delta 7.63(\mathrm{~s}, 1 \mathrm{H}), \delta 7.53(\mathrm{~s}, 1 \mathrm{H}), \delta 5.19(\mathrm{dd}, J 11.4 \mathrm{~Hz}, J 5.4 \mathrm{~Hz}, 1 \mathrm{H}), \delta 4.31-4.35(\mathrm{~m}, 2 \mathrm{H})$, $\delta$ 4.04-4.16 (m, 5H), $\delta 1.40(\mathrm{~s}, 9 \mathrm{H}), \delta 1.26(\mathrm{t}, J 7.2 \mathrm{~Hz}, 3 \mathrm{H}), \delta 1.23(\mathrm{~s}, 9 \mathrm{H}), \delta 1.17(\mathrm{t}, J 7.2 \mathrm{~Hz}$, $3 \mathrm{H}) .{ }^{13} \mathrm{C} \mathrm{NMR}\left(150 \mathrm{MHz}, \mathrm{CDCl}_{3}\right): \delta 193.5,183.8,158.7,143.4,138.2,136.2,131.8,131.1$, 130.3, 128.1, 121.4, 119.7, 63.2 (d, J $30 \mathrm{~Hz}), 47.0,44.4,43.5,35.0,34.4,31.2,29.6,16.2 .{ }^{31} \mathrm{P}$ NMR (243 MHz, $\left.\mathrm{CDCl}_{3}\right): \delta$ 12.6. ESI-MS: $m / z, 593[\mathrm{M}+\mathrm{H}]^{+}, 615[\mathrm{M}+\mathrm{Na}]^{+}, 631[\mathrm{M}+\mathrm{K}]$. Anal. calcd for $\mathrm{C}_{29} \mathrm{H}_{38} \mathrm{BrO}_{6} \mathrm{P}: \mathrm{C}, 58.69 ; \mathrm{H}, 6.45$. Found: C, 58.82; H, $6.51 \%$.

Synthesis of diethyl [2-(4-chlorophenyl)-1,4-dihydro-chromeno[4,3-b]pyrrole-3- phosphonate (4): To an oven-dried round-bottom flask equipped with a magnetic bar was added $\mathbf{1 a}$ (296 mg, $1.0 \mathrm{mmol}, 1.0$ equiv), pre-catalyst $3 \mathbf{a}$ (18 mg, $0.05 \mathrm{mmol}, 0.05$ equiv), 4-chlorobenzaldehyde (140 mg, $1.0 \mathrm{mmol}, 1.0$ equiv), anhydrous THF ( $2 \mathrm{ml}$ ), and dry $\mathrm{K}_{2} \mathrm{CO}_{3}$ ( $14 \mathrm{mg}, 0.1 \mathrm{mmol}, 0.10$ equiv.) under a $\mathrm{N}_{2}$ or $\mathrm{Ar}$ atmosphere at r.t. The resulting mixture was then stirred for $3 \mathrm{~h}$ at $15-17{ }^{\circ} \mathrm{C}$. The progress of the reaction was monitored by TLC. $\mathrm{NH}_{4} \mathrm{OAc}(231 \mathrm{mg}, 3 \mathrm{mmol})$ and gacial AcOH $(3 \mathrm{ml})$ were added under $\mathrm{N}_{2}$ atmosphere, the mixture was allowed to be stirred under reflux for $12 \mathrm{~h}$. Upon completion, the mixture was cooled to r.t. The workup involved addition of ice water $(20 \mathrm{ml})$ and extraction of the product mixture into $\mathrm{CHCl}_{3}(3 \times 20 \mathrm{ml})$, washing with sat. aq. $\mathrm{NaHCO}_{3}$ and sat. brine. After phase separation, drying over $\mathrm{Na}_{2} \mathrm{SO}_{4}$, filtration and evaporation, the crude product was purified by chromatography on silica using a 
mixture of petroleum ether and AcOEt (2:1) as an eluent to give 4 as white solid (208.5 mg, 50\%). Mp 106-107 ${ }^{\circ} \mathrm{C} .{ }^{1} \mathrm{H}$ NMR (600 MHz, $\left.\mathrm{CDCl}_{3}\right): \delta 10.33(\mathrm{~s}, 1 \mathrm{H}), \delta 7.50(\mathrm{~d}, J 7.8 \mathrm{~Hz}, 2 \mathrm{H}), \delta$ $7.39(\mathrm{~d}, J 7.2 \mathrm{~Hz}, 1 \mathrm{H}), \delta 7.18(\mathrm{~d}, J 7.2 \mathrm{~Hz}, 2 \mathrm{H}), \delta 7.07(\mathrm{t}, J 7.8 \mathrm{~Hz}, 1 \mathrm{H}), \delta 6.85-6.89(\mathrm{~m}, 2 \mathrm{H}), \delta$ $5.43(\mathrm{~s}, 2 \mathrm{H}), \delta 3.85-3.92(\mathrm{~m}, 4 \mathrm{H}), \delta 1.13(\mathrm{t}, J 7.2 \mathrm{~Hz}, 6 \mathrm{H}) .{ }^{13} \mathrm{C} \mathrm{NMR}\left(150 \mathrm{MHz}, \mathrm{CDCl}_{3}\right): \delta 158.4$, $156.5,149.3,138.4,136.5,135.7,134.1,131.7,129.8,128.5,126.2,122.7,122.2,116.7,66.5$, 62.6, 16.4. ${ }^{31} \mathrm{P}$ NMR (243 MHz, $\left.\mathrm{CDCl}_{3}\right): \delta$ 15.57. ESI-MS: $m / z, 418[\mathrm{M}+\mathrm{H}]^{+}, 440[\mathrm{M}+\mathrm{Na}]^{+}, 456$ $[\mathrm{M}+\mathrm{K}]$. Anal. calcd for $\mathrm{C}_{21} \mathrm{H}_{21} \mathrm{ClNO}_{4} \mathrm{P}: \mathrm{C}, 60.37$; H, 5.07; N, 3.35. Found: C, 60.24; H, 5.15; N $3.21 \%$.

\section{Acknowledgements}

This work was supported by the Natural Science Foundation of China (No. 20872046) and the Fundamental Research Funds for the Central Universities (No. CCNU09A02013).

\section{References}

1. For general review on domino reactions, see: (a) Tietze, L. F.; Brasche, G.; Gericke, K. Domino Reactions in Organic Synthesis; Wiley-VCH: Weinheim, 2006. http://dx.doi.org/10.1002/9783527609925

(b) Tietze, L. F. Chem. Rev. 1996, 96, 115. http://dx.doi.org/10.1021/cr950027e

PMid:11848746

(c) Guo, H.-C.; Ma, J.-A. Angew. Chem., Int. Ed. 2006, 45, 354. http://dx.doi.org/10.1002/anie.200500195

PMid:16287187

(d) Enders, D.; Grondal, C.; Hüttl, M. R. M. Angew. Chem., Int. Ed. 2007, 46, 1570. http://dx.doi.org/10.1002/anie.200603129

PMid:17225236

(e) Tietze, L. F.; Beifuss, U. Angew. Chem., Int. Ed. 1993, 32, 131.

http://dx.doi.org/10.1002/anie.199301313

2. (a) Stetter, H.; Kuhlmann, H. Synthesis 1975, 379

http://dx.doi.org/10.1055/s-1975-23759.

(b) Stetter, H.; Mohrmann, K.-H.; Schlenker, W. Chem. Ber. 1981, 114, 581. http://dx.doi.org/10.1002/cber.19811140218

3. El-Haji, T.; Martin, J. C.; Descotes, G. J. J. Heterocycl. Chem. 1983, 20, 233. http://dx.doi.org/10.1002/jhet.5570200148

4. Perrine, D. M.; Kagan, J.; Huang, D. B.; Zeng, K.; Theo, B. K. J. Org. Chem. 1987, 52, 2213. http://dx.doi.org/10.1021/jo00387a019 
5. Wynberg, H.; Metselaar, J. Synth. Commun. 1984, 14, 1. http://dx.doi.org/10.1080/00397918408060857

6. Jones, T. H.; Franko, J. B.; Blum, M. S.; Fales, H. M. Tetrahedron Lett. 1980, 21, 789. http://dx.doi.org/10.1016/S0040-4039(00)71506-1

7. For recent reviews on NHC catalysis, see:

(a) Enders, D.; Balensiefer, T. Acc. Chem. Res. 2004, 37, 534-541;

http://dx.doi.org/10.1021/ar030050j

PMid:15311952.

(b) Enders, D.; Niemeier, O.; Henseler, A. Chem. Rev. 2007, 107, 5606-5655;

http://dx.doi.org/10.1021/cr068372z

PMid:17956132.

(c) Marion, N.; Diéz-González, S.; Nolan, S. P. Angew. Chem. Int. Ed. 2007, 46, 2988-3000;

http://dx.doi.org/10.1002/anie.200603380.

PMid:17348057.

(d) Biju, A. T.; Kuhl, N.; Glorius, F. Acc. Chem. Res. 2011, 44, 1182-1195;

http://dx.doi.org/10.1021/ar2000716.

PMid:21751790. (e) Shen, L. T.; Shao, P. L.; Ye, S. Adv. Syn. \& Catal. 2011, 353, 1943-1948;

http://dx.doi.org/10.1002/adsc.201100178

(f) Bugaut, X.; Liu, F.; Glorius, F. J. Am. Chem. Soc. 2011, 133, 8130-8133;

http://dx.doi.org/10.1021/ja202594g PMid:21520959.

(g) Enders, D.; Grossmann, A.; Huang, H.; Raabe, G. Eur. J. Org. Chem. 2011, 4298-4301;

http://dx.doi.org/10.1002/ejoc.201100690

(h) Park, J. H.; Bhilare, S. V.; Youn, S. W. Org. Lett. 2011, 13, 2228-2231.

http://dx.doi.org/10.1021/ol200481u

PMid:21446687 (i) Grossmann, A.; Enders, D. Angew. Chem. Int. Ed. 2012, 51, 314-325.

http://dx.doi.org/10.1002/anie.201105415

PMid:22121084

8. Biju, A. T.; Wurz, N. E.; Glorius, F. J. Am. Chem. Soc., 2010, 132, 5970-5971.

http://dx.doi.org/10.1021/ja102130s

PMid:20384303

9. (a) Vedachalam, S.; Wong, Q. -L.; Maji, B.; Zeng, J.; Ma, J.; Liu, X. -W. Adv. Synth. Catal.

2011, 353, 219-225;

http://dx.doi.org/10.1002/adsc.201000828.

(b) Wang, Z. Q.; Yu, Z. H.; Wang, Y.; Shi, D. Q. Synthesis, 2012, 44, 1559-1568.

http://dx.doi.org/10.1055/s-0031-1290976

10. (a) Engel, R., Ed. Handbook of Organophosphorus Chemistry; Marcel Dekker: New York, 1992;

(b) Hartley, F. R., Ed. The Chemistry of Organophosphorus Compounds; John Wiley \& Sons:

New York, 1996; Vol. 4;

http://dx.doi.org/10.1002/0470034351 
PMid:9012996

(c) Quin, L. D., Ed. A Guide to Organophosphorus Chemistry; John Wiley \& Sons: New York, 2000; (d) Mader, M. M.; Bartlett, P. A. Chem. Rev. 1997, 97, 1281;

http://dx.doi.org/10.1021/cr960435y

PMid:11851452

(e) Brassfield, H. A.; Jacobson, R. A.; Verkade, J. G. J. Am.Chem. Soc. 1975, 97, 4143. http://dx.doi.org/10.1021/ja00847a053.

(f) Darrow, J. W.; Drueckhammer, D. G. J. Org. Chem. 1994, 59, 2976;

http://dx.doi.org/10.1021/jo00090a014.

(g) Cupta, H. C. L. Insecticides: Toxicology and Uses; Agrotech Publishing Academy Press:

Udaipur (India), 1999, p 51211;

(h) Matsumura, F. Toxicology of Insecticides, $2^{\text {nd }}$ ed.; New York: Plenum Press, 1985, pp 62-90.

http://dx.doi.org/10.1007/978-1-4613-2491-1

11. For the synthetic routes of NHC precursors, see:

(a) Pesch, J.; Harms, K.; Bach, T. Eur. J. Org. Chem. 2004, 2025-2035;

http://dx.doi.org/10.1002/ejoc.200300762

(b) Van Den Berg, H. J.; Challa, G.; Pandit, U. K. J. Molecular Catal. 1989, 51, 1-12;

http://dx.doi.org/10.1016/0304-5102(89)87003-8.

(c) Kerr, M. S.; Read de Alaniz, J.; Rovis, T. J. Org. Chem. 2005, 70, 5725-5728;

http://dx.doi.org/10.1021/jo050645n

PMid:15989360 PMCid:2527449.

(d) Arduengo, A. J.; Krafczyk, R.; Schmutzler, R.; Craig, H. A.; Goerlich, J. R.; Marshall, W.

J.; Unverzagt, M. Tetrahedron 1999, 55, 14523-14534.

http://dx.doi.org/10.1016/S0040-4020(99)00927-8

(e) Enders, D.; Gielen, H. J. Organomet. Chem. 2001, 617-618, 70-80;

http://dx.doi.org/10.1016/S0022-328X(00)00600-8

(f) Jazzar, R.; Liang, H. Z.; Donnadieu, B.; Bertrand, G. J. Organomet. Chem. 2006, 691, 3201-3205;

http://dx.doi.org/10.1016/j.jorganchem.2006.04.008

PMid:19543436 PMCid:2699275.

(g) Matsuoka, Y.; Ishida, Y.; Saigo, K. Tetrahedron Lett. 2008, 49, 2985-2989.

http://dx.doi.org/10.1016/j.tetlet.2008.02.175 